\title{
Silver nanoparticles with antimicrobial activities against Streptococcus mutans and their cytotoxic effect
}

\section{Authors: Mario Alberto Pérez-Díaz, Laura Boegli, Garth James, Cristina Velasquillo, Roberto Sánchez- Sánchez, Rita-Elizabeth Martínez-Martínez, Gabriel Alejandro Martínez-Castañón, \& Fidel Martinez- Gutierrez}

NOTICE: this is the author's version of a work that was accepted for publication in Materials Science and Engineering: C. Changes resulting from the publishing process, such as peer review, editing, corrections, structural formatting, and other quality control mechanisms may not be reflected in this document. Changes may have been made to this work since it was submitted for publication. A definitive version was subsequently published in Materials Science and Engineering: C, 55, October 2015. DOI\# http://dx.doi.org/10.1016/j.msec.2015.05.036.

Pérez-Díaz MA, Boegli L, James G, Velasquillo C, Sánchez-Sánchez R, Martínez-Martínez RE, Martínez-Castañón GA, Martinez-Gutierrez F, "Silver nanoparticles with antimicrobial activities against Streptococcus mutans and their cytotoxic effect," Materials Science and Engineering: C 55 (October 2015): 360-366. doi:10.1016/j.msec.2015.05.036. 


\title{
Silver nanoparticles with antimicrobial activities against Streptococcus mutans and their cytotoxic effect
}

\author{
Mario Alberto Pérez-Díaz ${ }^{a}$, Laura Boegli ${ }^{b}$, Garth James ${ }^{b}$, Cristina Velasquillo ${ }^{\mathrm{c}}$, Roberto \\ Sánchez-Sánchez ${ }^{\mathrm{c}}$, Rita-Elizabeth Martínez-Martínez ${ }^{\mathrm{d}}$, Gabriel Alejandro Martínez-Castañón \\ d, Fidel Martinez-Gutierrez ${ }^{\text {a, }}$ \\ a Facultad de Ciencias Químicas, UASLP, Álvaro Obregón 64, San Luis Potosí, Mexico \\ b Center for Biofilm Engineering, Montana State University, Bozeman, MT, USA \\ c Laboratorio de Biotecnología, Instituto Nacional de Rehabilitación, Mexico \\ d Facultad de Estomatología, Universidad Autónoma de San Luis Potosí, Mexico
}

\section{A b s t r a c t}

Microbial resistance represents a challenge for the scientific community to develop new bioactive compounds. The goal of this research was to evaluate the antimicrobial activity of silver nanoparticles (AgNPs) against a clin-ical isolate of Streptococcus mutans, antibiofilm activity against mature S. mutans biofilms and the compatibility with human fibroblasts. The antimicrobial activity of AgNPs against the planktonic clinical isolate was size and concentration dependent, with smaller AgNPs having a lower minimum inhibitory concentration. A reduction of $2.3 \mathrm{log}$ in the number of colony-forming units of S. mutans was observed when biofilms grown in a CDC reactor were exposed to $100 \mathrm{ppm}$ of AgNPs of $9.5 \pm 1.1 \mathrm{~nm}$. However, AgNPs at high concentrations (N10 ppm) showed a cytotoxic effect upon human dermal fibroblasts. AgNPs effectively inhibited the growth of a planktonic S. mutans clinical isolate and killed established S. mutans biofilms, which suggests that AgNPs could be used for prevention and treatment of dental caries. Further research and development are necessary to translate this technology into therapeutic and preventive strategies.

\section{Introduction}

Biofilms are microbial consortia embedded in self-produced exopoly-mer matrices composed of mainly exopolysaccharides (EPS). Microbes living in these matrices benefit from nutrient and water supplies [1], improved lateral gene transfer [2] and protection against adverse envi-ronmental insults, such as desiccation and chemicals, including deter-gents, disinfectants, and antibiotics [3,4]. Biofilms can be also reservoirs for pathogenic organisms and sources of disease outbreaks. For instance, biofilms are implicated in otitis media [5], otolaryngologic infections [6], osteomyelitis [7], bacterial endocarditis, cystic fibrosis [8], non-healing wounds [9] and oral biofilm [10].

Dental caries and periodontal diseases are widespread diseases, both of which are highly prevalent in industrialized societies, and are rising in developing countries [11]. Dental caries and periodontal diseases result from a complex interaction of environmental triggers, the resi-dent microorganisms and the host. If the composition of individual's resident oral microorganisms is out of balance, dental disease can occur [12]. Even at the earliest times of initial colonization, flowing sali-va bathes both cleaned surfaces and already attached cells with a variety of species suspended in saliva. A highly selective mechanism of coaggregation between species is involved in the development of mul-tispecies communities. The primary initial colonizers are streptococci and some Actinomyces, and early colonizing veillonellae coaggregate with streptococci and Actinomyces [13]. Although culture indepen-dent sequencing has illuminated the complexity of the human oral microbiome, Streptococcus mutans remains widely regarded as a pri-mary etiological agent in caries [14]. This facultative anaerobic, Gram positive, bacterium excels in the complex environment of the oral cavity where stresses including low $\mathrm{pH}$ and an oxidative environment are coupled to variable salivary flow and carbohydrate supply. The physio-logic adaptations of S. mutans to these pressures provide a competitive advantage versus non-cariogenic commensals that underpin the estab-lishment and progression of caries [15].

Treatment of an infection after the biofilm is established is frequently futile with current remedies. Often, the only solution is the physical removal of the biofilm or implant, which is costly and traumatic to the patient [16]. The control of oral biofilms depends in part on the use of chemical actives that kill or remove plaque. Actives that kill microor-ganisms presumably reduce bacterial virulence and retard the rate of plaque accumulation. The penetration of such actives into the microbial biofilm is a fundamental requirement for their efficacy [17].

In recent years the application of nanoparticles in various fields has been expanded considerably. Nanoparticles have been successfully 
used in medical and pharmaceutical nanoengineering for the delivery of therapeutic agents [18], in chronic disease diagnostics, and in sensors [19]. AgNPs are efficient non-specific antimicrobial agents against planktonic forms of a broad spectrum of bacterial and fungal species $[20,21]$. Their antimicrobial activities are attributed to the unique physicochemical characteristics of AgNPs, such as the high ratio of surface area to mass, high reactivity, and nanometer sizes, which confer them to a major advantage for the development of alternative products against multi-drug resistant microorganisms [22].

Knowledge of nanoparticle diffusivity is a parameter necessary to understand the mobility, aggregation, and toxicity of these composites. The diffusion of nanoparticles may be hindered by: (i) the porous structure of the biofilm; (ii) the local accumulation of nanoparticles by cells, non-diffusing macromolecules, or the polysaccharide matrix; and (iii) the adsorption of the solute to freely diffusing species, abiotic particles, or gas bubbles [23]. Due to the protection offered by the biofilm matrix to the diffusion of antibiotic agents within the exopolymer matrix, the antimicrobial activity of AgNPs was tested against both planktonic bacteria and biofilms formed under high fluid shear conditions using a bioreactor. Results presented in this study show that AgNPs were able to inhibit the growth of an S. mutans clinical isolate and also kill S. mutans inhabiting the biofilm matrix, suggesting that AgNPs could be used for the treatment of dental caries.

\section{Material and methods}

\subsection{Synthesis of AgNPs}

Silver nanoparticles with spherical and pseudospherical shapes with three different sizes were synthesized. All preparations started with a $0.01 \mathrm{M} \mathrm{AgNO}_{3}$ solution placed in a $250 \mathrm{ml}$ reaction vessel. Under magnetic stirring, $10 \mathrm{ml}$ of deionized water containing gallic acid $(0.1 \mathrm{~g}$ for 9.3 and $78.7 \mathrm{~nm}$ samples and $0.5 \mathrm{~g}$ for $21.3 \mathrm{~nm}$ sample) was added to $100 \mathrm{ml}$ of silver nitrate solution. After the addition of gallic acid the $\mathrm{pH}$ value of the solution was immediately adjusted (for the $9.5 \mathrm{~nm}$ sample the $\mathrm{pH}$ was raised to 11 with $\mathrm{NaOH} 1.0 \mathrm{M}$ and for the $25.9 \mathrm{~nm}$ sample, $\mathrm{pH}$ was raised to 10 with $\mathrm{NH}_{4} \mathrm{OH}$ ). For the $78.7 \mathrm{~nm}$ sample, after the addition of gallic acid, the mixture was irradiated with UV light ( $254 \mathrm{~nm}, 15 \mathrm{~W}$ ) for $30 \mathrm{~min}$ ( $\mathrm{pH}$ was not modified). After that, the solution was heated for $30 \mathrm{~min}$ at $80^{\circ} \mathrm{C}$ [24]. Immediately after the synthesis and in order to purify the silver nanoparticles, the obtained dispersions were dialyzed using a dialysis membrane ( $12 \mathrm{kDa}$ molecular weight) for $48 \mathrm{~h}$.

\subsection{Characterization of the AgNPs}

AgNPs were characterized by Dynamic Light Scattering (DLS), the hydrodynamic diameter and zeta potential were determined in triplicate by using a Malvern Zetasizer Nano ZS (Instruments Worcestershire, United Kingdom) operating with a He-Ne laser at a wavelength of $633 \mathrm{~nm}$, and a detection angle of $90^{\circ}$; all samples were analyzed for $60 \mathrm{~s}$ at $25^{\circ} \mathrm{C}$. To confirm shape, each sample was diluted with deionized water and $50 \mu \mathrm{l}$ of each suspension was placed on a copper grid for Transmission Electron Microscopy (TEM). All samples were analyzed by Transmission Electron Microscopy (JEOL JEM-1230, Tokyo, Japan) at an accelerating voltage of $100 \mathrm{kV}$.

\subsection{Patients}

The study included saliva from subjects with primary dentition, all residents of the city of San Luis Potosí, Mexico. Subjects were recruited by the department of Pediatric Dentistry of the Advanced General Dentistry Program. The children's parents completed a standardized health questionnaire that included oral pediatric information and assessments, as well as the recently used antibiotics. A voluntary informed consent was obtained from parents prior to the clinical examination, the research was done according to the Helsinki Declaration of 1975, and approved by the Masters Degree in Dental Science with Specialization in Advanced General Dentistry Program at San Luis Potosí University, Mexico. Pediatric samples of patients with active caries without any repair were considered. The inclusion criteria were children aged between 3 and 6 years. Exclusion criteria included those children who received antibiotics during the last three months preceding the survey.

\subsection{Sample collection}

Paraffin-stimulated whole saliva from children was sampled over a 5 min period in a sterilized propylene tube; this process was carried out consistently in the morning (9-10 am) to minimize the circadian rhythm effects, $2 \mathrm{~h}$ after the previous meal. Saliva samples were dispersed by sonication for $10 \mathrm{~s}$ and 10 -fold dilutions were prepared in saline solution $(0.9 \% \mathrm{NaCl}), 100 \mu \mathrm{l}$ of each dilution was spread by spiral bacteria plate (in duplicate) onto Mitis-salivarius agar (MSA) supplemented with $20 \%$ sucrose and $1 \%$ potassium tellurite. The plates were incubated in an atmosphere of $10 \% \mathrm{CO}_{2}$ at $37{ }^{\circ} \mathrm{C}$ for 24 hours (h); then plates were incubated for $24 \mathrm{~h}$ at room temperature. A stereoscopic microscope (Olympus, SD-ILK, Japan) was used to verify the presence of colony forming units (CFU) resembling $S$. mutans.

\subsection{Bacterial culture}

Microorganism strains were maintained in solidified broth using $1.5 \%$ agar trypticase soy plate supplemented with $5 \%$ sheep blood, cultures were placed in a humidified atmosphere supplemented with $5 \%$ $\mathrm{CO}_{2}$ for $24 \mathrm{~h}$ in stagnant condition. The clinical strain was identified using a VITEK $®$ system (data not shown).

\subsection{Antimicrobial test}

The microdilution method for estimation of minimum inhibitory concentration (MIC) values was carried out to evaluate the antimicrobial activity. The MIC values were determined on 96-well microdilution plates and according to published protocols [25]. MICs were determined by incubating $S$. mutans in 96-well microplates in a humidified atmosphere supplemented with $5 \% \mathrm{CO}_{2}$ for $24 \mathrm{~h}$. Microorganisms were exposed to serial dilutions of the nanoparticles, and the end points were determined when no turbidity in the well was observed. The antibacterial activities of the nanoparticles were compared to oxacillin. The turbidity background from the nanoparticles was subtracted from the final reading. All assays were carried out in triplicate.

\subsection{Biofilm formation on coupons}

Anti-biofilm activity of the $9.5 \mathrm{~nm}$ AgNPs was evaluated on high shear biofilm grown in the CDC Biofilm Reactor (CDC-BR) (model CBR 90, Biosurface Technologies Corporation, Bozeman, MT) [30] according to published protocols [26]. Briefly, the CDC-BR consists of a $1 \mathrm{~L}$ vessel with eight polypropylene coupon holders, which can accommodate three sample coupons ( 0.5 inch diameter) in each of the eight holders. Liquid growth medium enters through the top of the vessel and exits via a side-arm discharge port. A magnetic stir bar incorporating a mixing blade provides fluid mixing and surface shear creating a turbulent flow (Reynolds number between 800 and 1850). For these calculations, the bulk fluid was assumed to possess the properties of water at $20{ }^{\circ} \mathrm{C}$ when growing a $S$. mutans biofilm.

The reactor was filled with $400 \mathrm{ml}$ of $1 \%$ strength TSB and inoculated with $1 \mathrm{ml}$ of an overnight culture of $S$. mutans. The reactor was maintained in a batch mode (mixed, no flow) for $24 \mathrm{~h}$ at $37^{\circ} \mathrm{C}$. At the end of the first $24 \mathrm{~h}$ flow (1\% strength TSB) was started and maintained for another $24 \mathrm{~h}$. 


\subsection{Microscopic imaging of biofilms}

After biofilm formation in the CDC-BR, coupons were removed from the reactor, rinsed with $1 \mathrm{ml}$ of PBS and treated with $1 \mathrm{ml}$ of different sizes of AgNPs (9.5, 25.9 and $78.7 \mathrm{~nm}$ ) for $24 \mathrm{~h}$. Each coupon was fixed in glutaraldehyde and alcian blue of analytical grade for $24 \mathrm{~h} \mathrm{im-}$ mediately after removal. Subsequently, the coupons were dehydrated with ascending concentrations of ethanol. Completely processed
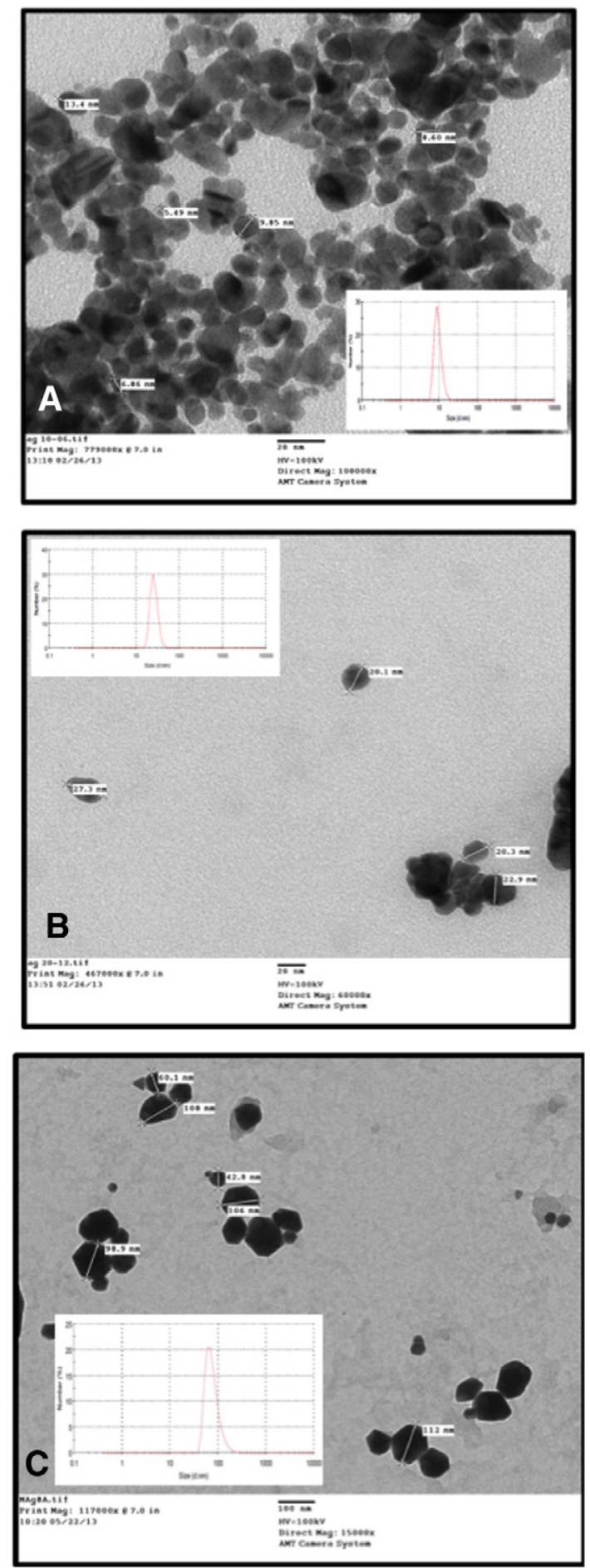

Fig. 1. Characteristics of nanoparticles used in this work. Transmission electron micrographs of silver nanoparticles at hydrodynamic size of (A) $9.5 \pm 1.1 \mathrm{~nm}$, (B) $25.9 \pm$ $1.1 \mathrm{~nm}$ and (C) $78.7 \pm 19.2 \mathrm{~nm}$. Inset: distribution of the nanoparticles according to size using Dynamic Light Scattering. samples were mounted separately on SEM disks, coated with gold, and examined with a scanning electron microscope (JEOL 6510HV) operated at an accelerating voltage of $15 \mathrm{kV}$, the study allowed us to know the dispersion of the AgNPs over the biofilm using a Backscattered electron detector (BSE). Captured images of the external surface of each coupon at WD of 8 to $11 \mathrm{~mm}$ were subsequently analyzed for biofilm; images obtained at higher magnifications were assessed only for the presence of bacterial cells and typical biofilm structure. The presence of biofilm was considered possible if a biofilm-like matrix was visible and if bacterial cell-like structures could be seen. The presence of bacterial cells was considered possible when cells consistent with cocci ( 0.5 to $1 \mu \mathrm{m}$ ) were visible and had at least one of the following characteristics: evidence of dividing cells or cell clustering consistent with microcolony formation.

\subsection{Colony counting}

After biofilm formation, coupons were removed from the reactor, rinsed with $1 \mathrm{ml}$ of PBS and treated with $1 \mathrm{ml}$ of different concentrations $(100,250,500$ and $1000 \mathrm{ppm})$ of AgNPs of size $9.5 \pm 1.1 \mathrm{~nm}$ for $24 \mathrm{~h}$. After the treatments, the coupons were rinsed again with $1 \mathrm{ml}$ of PBS and analyzed by plate counting. Three coupons from each treatment were used to quantify the number of bacterial cells. Coupons were removed and immersed in $9 \mathrm{ml}$ of sterile buffered saline solution. Biofilms were disaggregated using a sequence of treatments, which included vortexing (Vortex Genie 2; Scientific Products), sonication, and vortexing again alternating 120 second cycles of sonication at a frequency of $42 \mathrm{kHz}$ (model 2510 sonicating water bath; Branson), followed by a 30 second vortexing and according to published protocols [29]. The biofilm suspensions obtained after the treatments were serially diluted in sterile buffered saline solution, plated in triplicate on the corresponding plates, and incubated under conditions mentioned above. The CFU were counted after $18 \mathrm{~h}$ of incubation.

\subsection{Viability evaluation}

In order to know the behavior of silver nanoparticles on human fibroblasts, a cell viability assay was performed. The consent and experimental protocols in this study were reviewed and approved by the ethics committee of Instituto Nacional de Rehabilitacion (México, D.F.). Fibroblasts were obtained from circumcision surgeries from pediatric foreskin (with informed consent), the epidermis was separated from the dermis using dispase (SIGMA) for $8 \mathrm{~h}$; the dermis was treated for $4 \mathrm{~h}$ with collagenase I (Worthington Biochemical) to obtain the fibroblasts. Cells were cultured with DMEM-F12 medium (GIBCO) supplemented with 10\% BFS (GIBCO) and 1\% penicillin/streptomycin (GIBCO), and maintained in an incubator at $37{ }^{\circ} \mathrm{C}, 83 \%$ humidity and $5 \% \mathrm{CO}_{2}$. Fibroblasts used in the assays were passage 3 . Viability of human dermal fibroblast was evaluated using a Live/Dead viability/ cytotoxicity kit for mammalian cells (Molecular probes, Invitrogen). Cells were seeded at a $30,000 \mathrm{cell} / \mathrm{cm}^{2}$ density over 24 well culture dishes. Cell were exposed to $9.5 \mathrm{~nm}$ AgNPs at concentrations of 10, 50, 100, 150 and 200 ppm for 24 h. Cells were washed twice with a

Table 1

Characteristics of the silver nanoparticles used in this work

\begin{tabular}{|c|c|c|c|c|}
\hline $\begin{array}{l}\text { Hydrodynamic } \\
\text { diameter }^{\mathrm{a}} \\
\text { DLS }(\mathrm{nm})\end{array}$ & $\begin{array}{l}\text { Diameter }^{\mathrm{a}} \\
\text { TEM (nm) }\end{array}$ & Shape & $\begin{array}{l}\text { Concentration } \\
(\mathrm{ppm})\end{array}$ & $\begin{array}{l}\text { Zeta } \\
\text { potential }^{\mathrm{b}} \\
(\mathrm{mV})\end{array}$ \\
\hline $9.5 \pm 1.1$ & $10.0 \pm 2.66$ & Spherical & 1070 & $-5.83 \pm 3.18$ \\
\hline $25.9 \pm 2.6$ & $20.2 \pm 3.28$ & Spherical & 1070 & $-52.6 \pm 4.25$ \\
\hline $78.7 \pm 19.2$ & $77.9 \pm 27.54$ & Pseudospherical & 1070 & $-46.3 \pm 3.72$ \\
\hline
\end{tabular}

DLS: Dynamic Light Scattering. TEM: Transmission Electron Microscopy.

a Diameters are expressed in average and standard deviation.

b Zeta potential is expressed in average and standard deviation. 
Table 2

Antimicrobial activities showed against planktonic form of S. mutans

\begin{tabular}{llcll}
\hline \multicolumn{4}{l}{ Antimicrobials } & \\
\cline { 2 - 4 } & AgNPs & & Commercial \\
\hline Size (nm) & $9.5 \pm 1.1$ & $25.9 \pm 2.6$ & $78.7 \pm 19.2$ & Oxacillin \\
MIC (MIC (iiii) $)^{\text {b }}$ & $4.0 \pm 0$ & $8.0 \pm 0$ & $16.0 \pm 0$ & $16.0 \pm 0$ \\
\hline
\end{tabular}

MIC, minimum inhibitory concentration.

Size is expressed in average and standard deviation.

b MIC is expressed in average and standard deviation.

phosphate buffered saline (PBS) and incubated for 45 min in Hank's balanced salt solution (HBSS; GIBCO) with $2 \mu \mathrm{M}$ calcein AM and $2 \mu \mathrm{M}$ ethidium homodimer (EthD-1). Calcein fluorescent signal was observed with a Fluorescein band pass filter and EthD-1 with a Texas red filter. Images were captured and analyzed using an Axiovision Observer A.1 microscope (Zeiss)

\subsection{Statistical analysis}

The density recorded for each coupon was log10-transformed. All statistical calculations were performed on the log density values. For each test, the log densities were converted into a log reduction measure of efficacy. The log reduction is the mean log density for control coupons or membranes minus the mean log density for the corresponding treated coupons [27]. Statistical analysis for significance was determined using a two-tailed $t$-test assuming unequal variances with $\alpha=0.5$ and a $P$ value $\leq 0.05$ was considered to be significant.

For cell viability analysis the cells were counted using Image J software, the percentage of live and dead cells was determined, and ANOVA and the Tukey test were performed for this assay.

\section{Results}

\subsection{Synthesis and characterization of the AgNPS}

TEM revealed that AgNPs were of spherical and pseudospherical shapes. The nanoparticles synthesized in aqueous solution showed a narrow size distribution according to measures by DLS, also the hydrodynamic diameter $( \pm S D$ ) of the AgNPs was $9.5 \pm 1.1$ (Fig. 1A), $25.9 \pm$ 2.6 (Fig. 1B) and $78.7 \pm 19.2 \mathrm{~nm}$ (Fig. 1C), and the zeta potential values range from $-5.83 \pm 3.18$ to $-52.6 \pm 4.25 \mathrm{mV}$ (Table 1 ).

\subsection{Samples and bacterial strains}

From the samples of saliva, from pediatric patients, the microbiological analysis showed that the isolated clinical pathogenic strains commonly found were associated with a primary etiological agent in caries. Strains isolated in agar culture plate presented specific morphology characteristics, showed that $S$. mutans were the more prevalent, the biochemical identification was performed by VITEK $®$ and the confirmation of the serotype was carried out using PCR, the results of high prevalence of $S$. mutans serotype c was in correlation with the previous results reported by Espinosa-Cristóbal et al. in saliva samples of children in our region [28].

\subsection{Antimicrobial test}

The AgNPs showed antimicrobial activity whit size and concentration behavior, like previously was reported the antimicrobial activity of AgNPs against planktonic form of different microorganisms showed better results with the smallest [29]. Here, the evaluation of the antimicrobial activity of AgNPs of size $9.5+1.1 \mathrm{~nm}$ against the planktonic form of clinical strain of S. mutans showed the highest effect, with
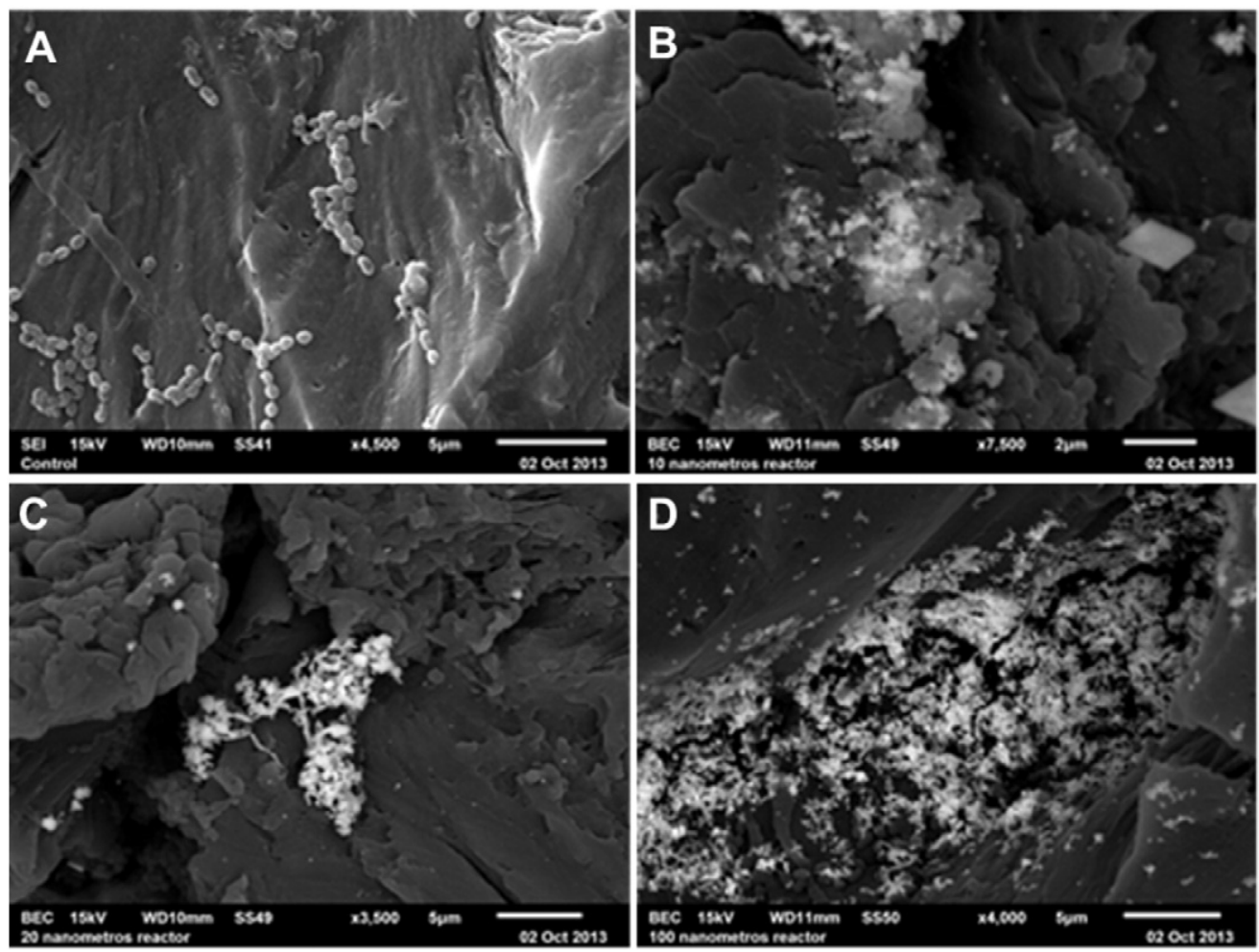

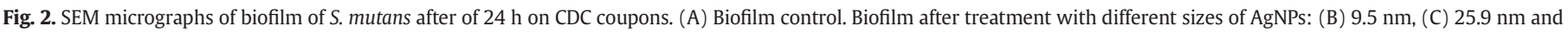
(D) $166.5 \mathrm{~nm}$. The BSE image shows the AgNPs with bright contrast. 

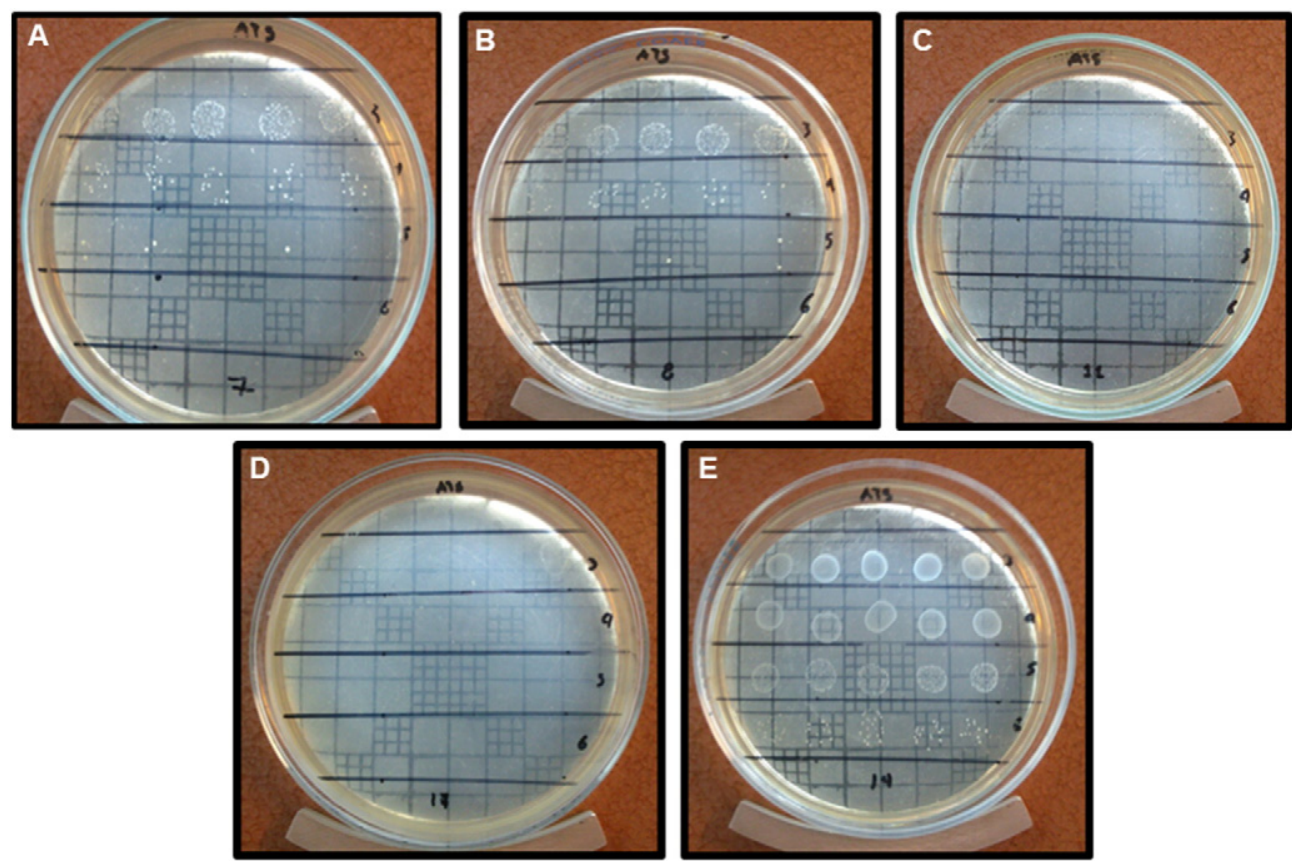

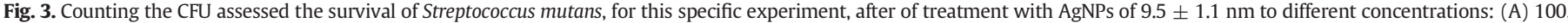
(B) 250, (C) 500 and (D) 1000 ppm, (E) control without AgNPs. Experiments were performed in triplicate.

MIC averages of $4 \pm 0 \mathrm{ppm}$, which was better than that achieved with commercial antibiotic oxacillin, with an MIC average of $16 \pm 0 \mathrm{ppm}$. The AgNPs with sizes 25.9 and $78.7 \mathrm{~nm}$ showed an MIC average of 8 and 16 ppm respectively (Table 2 ).

\subsection{Microscopic imaging of biofilms}

Mature S. mutans biofilms and individual cells were observed with SEM, the homogeneous structure of extracellular polymeric substance (EPS) after of the dehydration process was observed in the control without AgNP treatment (Fig. 2A), considering that EPS is such an integral component of the biofilm and it is a crucial target for biofilm disruption experimentation. It was possible to distinguish the contrast difference between $\mathrm{Ag}$ and biofilm structure due to their atomic number difference, when the Backscattered electron detector (BSE) was used. The distribution of the AgNPs of different sizes (Fig. 2B, C and D), on mature biofilm was homogeneous, and the structure of EPS was altered for the treatment with the AgNPs in comparison with the control. Fewer AgNPs were observed on the biofilm when the smallest AgNPs were used, compared to the largest AgNPs.

\subsection{Colony counting}

To determine the anti-biofilm activity of AgNPs, mature S. mutans biofilms were grown on hydroxyapatite coupons in CDC-BR [30]. After $24 \mathrm{~h}$ of growth, high cell density biofilms were observed on the coupons, the coupons were treated with different concentrations (100, 250, 500 and $1000 \mathrm{ppm}$ ) of AgNPs of size $9.5 \pm 1.1 \mathrm{~nm}$ (Fig. 3A, B, C and D), and at the same time, coupons were treated with PBS like control of growth (Fig. 3E), which showed the highest growth, all the treatments were evaluated by viable plate counts.

Concentrations as low as $100 \mathrm{ppm}$ of AgNPs showed a $>2.3-\log$ reduction, while increasing the AgNP concentration up to $1000 \mathrm{ppm}$ resulted in a 7-log reduction (Fig. 4).

\subsection{Cell viability analysis}

For the analysis of viability we chose the AgNPs of size $9.5 \pm$ $1.1 \mathrm{~nm}$, because they showed significant activity when tested against the planktonic form, which was better than that achieved with commercial antibiotic oxacillin, as well as, the results of the biofilm structure treated with AgNPs of different sizes, AgNPs of size $9.5 \pm 1.1 \mathrm{~nm}$ showed changes at the EPS.

Viability tests with ethidium homodimer and calcein, revealed that increasing doses of silver nanoparticles killed human dermal fibroblasts as shown in the graph of cell density (Fig. 5). AgNPs (9.5 $\pm 1.1 \mathrm{~nm}$ ) were tested at concentrations of $10,50,100,150$ and $200 \mathrm{ppm}$ in fibroblast medium. The percentages of live and dead cells were determined after $24 \mathrm{~h}$ of the cells being in contact with AgNPs. At a concentration of $10 \mathrm{ppm}$ AgNPs did not affect fibroblast viability, but at a concentration of 50 ppm cell viability decreased by $50 \%$ and at 200 ppm most of the fibroblasts were dead, a classic dose response curve.

\section{Discussion}

Studies of NPs as antibacterial, antifouling, and antibiofilm agents have increased, and over the last few years their incorporation in new

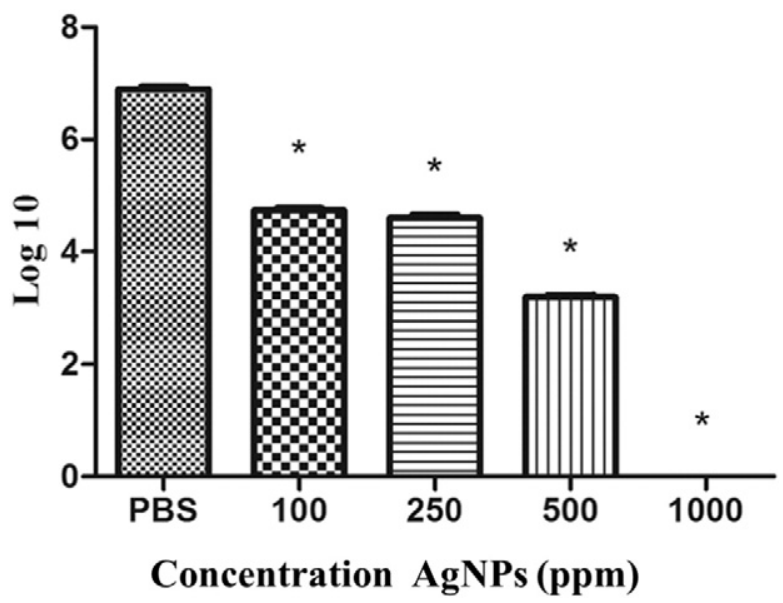

Fig. 4. Antimicrobial activities of AgNPs against biofilms formed in the CDC reactor. The log reduction was calculated relative to untreated control biofilms. The mean $\pm \mathrm{SD}$ of three independent experiments is shown. ${ }^{*}=P$ value $\leq 0.05$. 

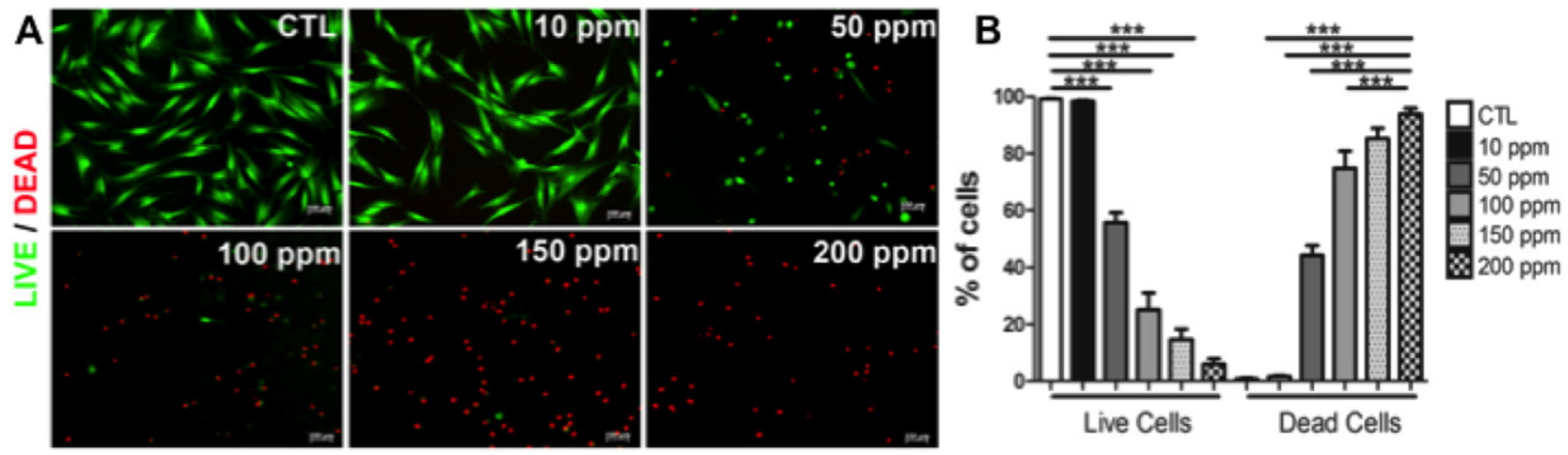

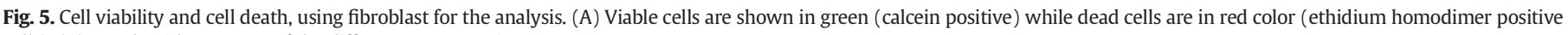
cells). (B) Graph with response of the different concentrations.

composites has been escalating. A sufficient variety of biofilm models are now commercially available or may be constructed in the laboratory. One of the main issues for uninitiated researchers is making a rational choice regarding the best model to use. Generally, systems that closely reproduce in situ conditions should be chosen when the aim is solely to reproduce natural biofilms under laboratory conditions. The use of a CDC reactor is favored because of its standard protocol [2], which has been systematically evaluated. When run according to standard procedures the CDC-BR has shown to be reliable and is relatively insensitive to minor perturbations in the time allowed for initial surface colonization, culture temperature, nutrient concentration and fluid shear stress [30], in fact, it provides reproducible biofilm samples under consistent growth conditions for the evaluation of antimicrobial agents and surface materials.

In the context of application of the nanotechnology to biological area, one study with a bonding agent containing nanoparticles of silver developing a resin-mica sandwich reveled that AgNP-containing adhesive had a long-distance killing capability and inhibited bacteria on its surface and away from its surface in a 24-well plate model [31]. Here we report a MIC of $4 \mathrm{ppm}$ and $2.3 \mathrm{log}$ reduction of $S$. mutans biofilms from $9.5 \pm 1.1 \mathrm{~nm}$ AgNPs at concentrations $\geq 100 \mathrm{ppm}$. Care is required when interpreting data derived from this type of model, since previous research has demonstrated that biofilm grown under high fluid shear conditions, CDC-BR, produced the smallest LR and biofilm grown in the absence of fluid shear produced the largest LR [32,33]. These results are in line with previous studies reporting that biofilms grown in turbulent flow conditions showed more mass resulting in an increase of the density, physiological activity, and total protein when compared to biofilms generated under static conditions [32].

AgNPs' effect on biofilm may be due to the interference or inhibition of the bacterial machinery that controls the internal production and/or regulation of EPS. The mechanism of deactivation of proteins can be explained by the reaction of $\mathrm{Ag}^{+}$ions with cysteine residues present in proteins as demonstrated in a previous study which exposed the human hepatoma HepG2 cells to N-acetyl-cysteine (NAC), an antioxidant and glutathione precursor prior to AgNP exposure. Results of this study showed that NAC treatment was equivalent to untreated controls [20]. Then, as a result of an ionization of the AgNPs, $\mathrm{Ag}^{+}$ions can interact with protein and enzyme thiol groups, such as cysteines with further damage [34]. Results of the present study show that AgNPs kill the biofilm-associated bacteria. Further studies are needed to investigate the role of $\mathrm{Ag}^{+}$ions in AgNP toxicity and diffusion through the biofilm. To examine this bactericidal activity, higher concentrations of AgNPs (ranging between 100 and 1000 times) are necessary to kill microbes within biofilms as compared to concentrations needed to kill planktonic forms. These results agree with the previous work of Ashkarran AA et al. [35], which concluded that AgNPs should have dual toxicity effects (high toxic effect on bacteria and no/low toxic (biocompatible) effect on human cells). This investigation shows that functionalized AgNPs covering the surfaces would provide great potential for the prevention and treatment of infections related to biofilm formation. However, further research and development are necessary to translate this technology into therapeutic and preventive strategies.

\section{Conclusions}

AgNPs effectively prevent biofilm formation and kill bacteria in established biofilms, which suggests that they could be embedded into the matrices or material used for the fabrication of medical devices to avoid adherence, colonization and biofilm formation of microorganisms. Further research and development are necessary to translate this technology into therapeutic and preventive strategies.

\section{Acknowledgments}

The funding for these studies was provided by: PROMEP 103.5/12/ 7965, folio UASLP-EXB-213 and scholarships from Sistema Nacional de Investigadores (SNI 48199) and Consejo Nacional de Ciencia y Tecnología (CONACyT): 233241. We thank Drs. J. William Costerton and Philip S. Stewart for their support in this project.

\section{References}

[1] C.C. Goller, T. Romeo, Environmental influences on biofilm development, Curr. Top. Microbiol. Immunol. 322 (2008) 37-66.

[2] T.F. Maha, G.A. O'Toole, Mechanisms of biofilm resistance to antimicrobial agents, Trends Microbiol. 9 (2001) 34-39.

[3] P.S. Stewart, J.W. Costerton, Antibiotic resistance of bacteria in biofilms, Lancet 358 (2001) 135-138

[4] J.W. Costerton, Bacterial attachment to surfaces, in: D.C. Eckey (Ed.), The Biofilm Primer, Springer, Berlin 2007, pp. 36-43.

[5] L.O. Bakaletz Bakaletz, Bacterial biofilms in otitis media: evidence and relevance, Pediatr. Infect. Dis. J. 26 (2007) S17-S19.

[6] J.C. Post, N.L. Hiller, L. Nistico, P. Stoodley, G.D. Ehrlich, The role of biofilms in otolaryngologic infections, Curr. Opin. Otolaryngol. Head Neck Surg. 15 (2007) 347-351.

[7] R.A. Brady, J.G. Leid, J.H. Calhoun, J.W. Costerton, M.E. Shirtliff, Osteomyelitis and the role of biofilms in chronic infection, FEMS Immunol. Med. Microbiol. 52 (2008) $13-22$.

[8] N. Høiby, Understanding bacterial biofilms in patients with cystic fibrosis: current and innovative approaches to potential therapies, J. Cyst. Fibros. 1 (2002) 249-254.

[9] G.A. James, E. Swogger, R. Wolcott, E. Pulcini, P. Secor, J. Sestrich, J.W. Costerton, P.S. Stewart, Biofilms in chronic wounds, Wound Repair Regen. 16 (2008) 37-44.

[10] P.E. Kolenbrander, J. London, Adhere today, here tomorrow: oral bacterial adherence, J. Bacteriol. 175 (11) (1993) 3247-3252.

[11] R.A. Bagramian, F. Garcia-Godoy, A.R. Volpe, The global increase in dental caries. A pending public health crisis, Am. J. Dent. 22 (1) (2009) 3-8.

[12] R.J. Palmer Jr., Composition and development of oral bacterial communities, Periodontol. 64 (1) (2014) 20-39 (2000).

[13] P.E. Kolenbrander, R.J. Palmer Jr., S. Periasamy, N.S. Jakubovics, Oral multispecies biofilm development and the key role of cell-cell distance, Nat. Rev. Microbiol. 8 (7) (2010) 471-480.

[14] F.E. Dewhirst, T. Chen, J. Izard, B.J. Paster, A.C. Tanner, W.H. Yu, A. Lakshmanan, W.G. Wade, The human oral microbiome, J. Bacteriol. 192 (2010) 5002-5017.

[15] J.A. Lemos, R.G. Quivey Jr., H. Koo, J. Abranches, Streptococcus mutans: a new Grampositive paradigm? Microbiology 159 (Pt 3) (2013) 436-445. 
[16] A.S. Lynch, G.T. Robertson, Bacterial and fungal biofilm infections, Ann. Rev. Med. 59 (2008) 415-428.

[17] A. Corbin, B. Pitts, A. Parker, P.S. Stewart, Antimicrobial penetration and efficacy in an in vitro oral biofilm model, Antimicrob. Agents Chemother. 55 (7) (2011) 3338-3344.

[18] L. Zhang, F.X. Gu, J.M. Chan, A.Z. Wang, R.S. Langer, O.C. Farokhzad, Nanoparticles in medicine: therapeutic applications and developments, Clin. Pharmacol. Ther. 83 (2008) 761-769.

[19] B. Hong, J. Kai, Y. Ren, J. Han, Z. Zou, C.H. Ahn, Highly sensitive rapid, reliable, and automatic cardiovascular disease diagnosis with nanoparticle fluorescence enhancer and MEMS, Adv. Exp. Med. Biol. 614 (2008) 265-273.

[20] S. Kim, J.E. Choi, J. Choi, K.H. Chung, K. Park, J. Yi, D.Y. Ryu, Oxidative stressdependent toxicity of silver nanoparticles in human hepatoma cells, Toxicol. In Vitro 23 (2009) 1076-1084.

[21] F. Martínez-Gutierrez, P.L. Olive, A. Banuelos, E. Orrantia, N. Nino, E. Morales Sanchez, et al., Synthesis, characterization, and evaluation of antimicrobial and cytotoxic effect of silver and titanium nanoparticles, Nanomed. Nanotechnol. Biol. Med. 6 (2010) 681-688.

[22] F. Martínez-Gutierrez, E.P. Thi, J.M. Silverman, C.C. de Oliveira, S.L. Svensson, A. Vanden Hoek, E.M. Sánchez, N.E. Reiner, E.C. Gaynor, E.L. Pryzdial, E.M. Conway, E. Orrantia, F. Ruiz, Y. Av-Gay, H. Bach, Antibacterial activity, inflammatory response, coagulation and cytotoxicity effects of silver nanoparticles, Nanomed. Nanotechnol. Biol. Med. 8 (2012) 328-336.

[23] P.S. Stewart, Diffusion in biofilms, J. Bacteriol. 185 (2003) 1485-1491.

[24] L.F. Espinosa-Cristóbal, G.A. Martínez-Castañón, R.E. Martínez-Martínez, J.P. LoyolaRodríguez, N. Patiño-Marín, J.F. Reyes-Macías, F. Ruiz, Antibacterial effect of silver nanoparticles against Streptococcus mutans, Mater. Lett. (2009) 2603-2606.

[25] Clinical and Laboratory Standards Institute, Methods for Dilution Antimicrobial Susceptibility Tests for Bacteria that Grow Aerobically: Approved Standard Seventh Edition M7-A7, vol. 26(2), Clinical and Laboratory Standards Institute, Wayne, PA, 2006.
[26] ASTM International, E-2562-07: standard test method for quantification of a Pseudomonas aeruginosa biofilm grown with shear and continuous flow using a rotating disk reactor, Annual Book of ASTM Standards, vol. 11.05, ASTM International, West Conshohocken, PA, 2007.

[27] N. Zelver, M. Hamilton, D. Goeres, J. Heersink, Development of a standardized antibiofilm test, Methods Enzymol. 337 (2001) 363-376.

[28] L.F. Espinosa-Cristóbal, G.A. Martínez-Castañón, J.P. Loyola-Rodríguez, J.F. ReyesMacías, F. Ruiz, Antimicrobial sensibility of Streptococcus mutans serotypes to silver nanoparticles, Mater. Sci. Eng. C Mater. Biol. Appl. (2012) 896-901.

[29] G.A. Martínez-Castañón, N. Niño-Martínez, F. Martínez-Gutierrez, J.R. MartínezMendoza, F. Ruiz, Synthesis and antibacterial activity of silver nanoparticles with different sizes, J. Nanoparticle Res. 10 (2008) 1343-1348.

[30] D.M. Goeres, L.R. Loetterle, M.A. Hamilton, R. Murga, D.W. Kirby, R.M. Donlan, Statistical assessment of a laboratory method for growing biofilms, Microbiology 151 (2005) 757-762.

[31] F. Li, M.D. Weir, J. Chen, H.H. Xu, Comparison of quaternary ammonium-containing with nano-silver-containing adhesive in antibacterial properties and cytotoxicity, Dent. Mater. 4 (2013) 450-461.

[32] K. Buckingham-Meyer, D.M. Goeres, M.A. Hamilton, Comparative evaluation of biofilm disinfectant efficacy tests, J. Microbiol. Methods 70 (2007) 236-244.

[33] I.W. Sutherland, The biofilm matrix an immobilized but dynamic microbial environment, Trends Microbiol. 9 (5) (2001) 222-227.

[34] J.L. Elechiguerra, J.L. Burt, J.R. Morones, A. Camacho-Bragado, X. Gao, H.H. Lara, M.J. Yacaman, Interaction of silver nanoparticles with HIV-1, J. Nanobiotechnol. 29 (2005) 3-6.

[35] A.A. Ashkarran, M. Ghavami, H. Aghaverdi, P. Stroeve, M. Mahmoudi, Bacterial effects and protein corona evaluations: crucial ignored factors in the prediction of bio-efficacy of various forms of silver nanoparticles, Chem. Res. Toxicol. 6 (2012) 1231-1242. 